\title{
Valve-sparing aortic root replacement in a patient with a rare connective tissue disorder: Arterial tortuosity syndrome
}

\author{
Tomaso Bottio, MD, PhD, Gianluigi Bisleri, MD, Paolo Piccoli, MD, and Claudio Muneretto, MD, Brescia, Italy
}

B Supplemental material is available online.

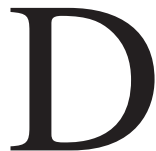
ilation of the aortic root and the ascending aorta is a well-known cardiovascular manifestation in pediatric patients with underlying connective tissue disorders, ${ }^{\mathrm{E} 1}$ often leading to aortic valve incompetence and to aortic dissection risk. The Bentall procedure, a preventive operation, is a low-risk operation with considerably durable outcomes. ${ }^{1}$ Alternatively, other more conservative techniques have been proposed: aortic root remodeling and reimplantation. ${ }^{2,3}$

Arterial tortuosity syndrome (ATS) is a rare condition with an autosomal recessive inheritance characterized by tortuosity, elongation, stenosis, and aneurysm formation in the major arteries. ${ }^{4}$ Approximately 35 patients from 21 families have been reported thus far worldwide. This is the first case ever reported in the literature of a patient with ATS undergoing aortic root surgery with sparing of the aortic valve.

\section{Clinical Summary}

The patient, a 19-year-old man, was of a consanguineous family. One older sibling had ATS, as well as 2 of 3 cousins. ${ }^{4}$ At birth, he experienced a cardiac arrest. Dilation and elongation of the ascending aorta and arch was observed, followed by abrupt narrowing of the descending thoracic aorta; pulmonary arteries were aneurysmal and tortuous. A mutation in a third member of the SLC2A family (SLC2A10) was observed in this child. According to clinical features and genetic mapping, diagnosis of ATS was accomplished. ${ }^{4}$ In February 2004, the aortic annulus, the sinus portion of the ascending aorta, the sinotubular junction, and the ascending aorta measured $25,38,27$, and $30 \mathrm{~mm}$, respectively. At the following examination, in January 2005, a progressive dilation was observed in the sinus portion and sinotubular junction (44 mm and $29 \mathrm{~mm}$, respectively). In March 2005, the patient had recurrent episodes of thoracic pain. A new echocardiogram revealed a further aortic sinus dilation, from 44 to $48 \mathrm{~mm}$, with a moderate aortic valve incompetence (Figure 1). Aortic angiography and computed

\footnotetext{
From the Department of Cardiovascular Surgery, University of Brescia Medical School, Brescia, Italy.

Received for publication June 23, 2006; accepted for publication Aug 25, 2006.

Address for reprints: Tomaso Bottio, MD, Department of Cardiovascular Surgery, University of Brescia Medical School, Piazza Spedali Civili, 1, Brescia, Italy 25100 (E-mail: bottio@med.unibs.it).

J Thorac Cardiovasc Surg 2007;133:252-3

$0022-5223 / \$ 32.00$

Copyright $\odot 2007$ by The American Association for Thoracic Surgery doi:10.1016/j.jtcvs.2006.08.050
}

tomography (16 detectors, Figure E1) confirmed the echocardiographic data. At Doppler ultrasonography, an extreme narrowing and tortuosity of the arteries was observed. In light of moderate aortic valve incompetence with normal aortic valve leaflets, a valve-sparing aortic root replacement procedure (Tirone David I technique) was performed.

The patient was discharged home free of cardiac and breathing symptoms 8 days after the procedure on oral antiplatelet treatment. One year later, the patient is well and living at home. Aortic valve function is perfect at 1-year echocardiographic follow-up. Histology of the aortic wall was performed (Figure E2).

\section{Discussion}

We describe a patient with aortic root dilation subordinate to an inherited uncommon connective tissue disorder characterized by elastic unit disruption in the median layer of the arterial wall (ATS) and the surgical procedure that we adopted to prevent further aortic dilation, rupture, or both while preserving the aortic valve.

ATS is an autosomal recessive connective tissue disorder recognized to be caused by loss-of-function mutations in the third member of the SLC2A family (SLC2A10). ${ }^{4}$ Defects in the elastic collagen network are also found in other connective tissue disorders having overlapping phenotypic and arterial features with ATS, such as Loeys-Dietz syndrome (LDS), ${ }^{5}$ and other wellknown syndromes. ${ }^{3, \mathrm{E} 1}$ Nevertheless, the ATS and LDS pathophysiology are substantially divergent. LDS is caused by heterozygous loss-of-function mutations in the genes encoding the type 1 or type 2 TGFB receptors. These primary alterations in the transforming growth factor beta (TGFB) receptors lead to increased TGFB signaling in the arterial wall. Similarly, patients with ATS show a TGFB activation, but the mechanism is unclear. Recently, Coucke and colleagues ${ }^{4}$ analyzed the expression of decorin in cultured vascular smooth muscle cells of individuals with LDS and those with ATS. The decorin is a proteoglycan inhibitor of TGFB signaling, and its expression was severely reduced in patients with ATS compared with that seen in patients with LDS. Therefore, the specific decrease of decorin expression in individuals with ATS might indicate a divergent mechanism for upregulation of TGFB signaling in ATS compared with LDS. In this child the primary alterations in the TGFB receptors, typical of LDS syndrome, were not observed. Meanwhile, the decorin mechanism has been verified. ${ }^{4}$ The more striking lesion is the degree of arterial tortuosity and lengthening associated with aneurysms and stenoses confined to arterial vessels, which is much more severe than in other more common connective tissue disorders (Marfan syndrome and Ehlers-Danlos syndrome). In such cases ascending aortic dilation usually is clinically evident with a time-dependent progression, often leading to aortic valve incompetence.

The elastic fibrils of a normal ascending aorta are long and run parallel with one another in an orderly fashion. ${ }^{\mathrm{E} 2}$ On the contrary, 

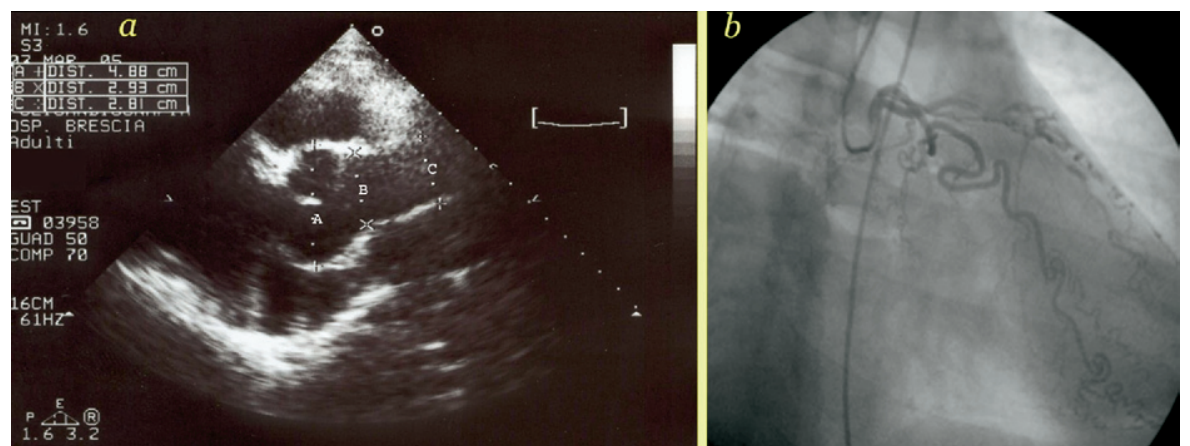

Figure 1. a, Two-dimensional echocardiogram. Note the aortic sinus dilation. b, Coronary angiogram shows an extreme tortuosity of the left anterior descending. The coronary artery origin and course were normal.

because elastic lamellae in the aorta of patients with ATS are fewer, short, and stick-like structures, affected patients are exposed to an increased risk of aortic complications. The Bentall procedure allows a complete removal of pathologic aortic wall, including also the valve leaflets. Nevertheless, it is burdened by a lifelong risk of anticoagulant-related events. In light of these drawbacks, valvesparing aortic root replacement has gained popularity. Remodelingtype $^{3}$ or reimplantation-type ${ }^{2}$ techniques are safe and reproducible, guaranteeing satisfactory long-term results. It is still debated what procedure should be preferred; nevertheless, a lower long-term freedom from recurrent aortic valve incompetence after the remodeling type has been reported, mostly for patients with a connective tissue disorder. ${ }^{2,3}$

We infer that these different long-term outcomes might find an explanation in the aortic root histology. On the sinus aortic wall, the elastic lamellae continue along the luminal side, with collagen being situated at the outside. On the contrary, the interleaflet triangle, the aortic root area between the ventricular myocardium and valve commissures, consists of collagen tissue on the inside and elastic lamellae on the outside. ${ }^{\mathrm{E} 3}$ Therefore even the interleaflet triangles are compromised in the presence of elastic unit disruption.

Considering the ATS features, we suggest the reimplantation technique because such a strategy allows replacement of the entire pathologic aortic root to reinforce the aortic annulus, including the valve and the pathologic interleaflet triangles within the graft, to add a fundamental structural support to this particularly fragile aortic area. Otherwise, in case of remodeling aortic root replacement, the interleaflet triangles are exposed directly and without any support to the left ventricular outflow tract, leading to a major risk of dilation. Preserving the aortic valve and avoiding chronic oral anticoagulation, this technique is hardly indicated in patients with ATS in whom the cerebral hemorrhage risk is very high.

In summary, this case, unique in the literature thus far, adds new concepts in favor of the reimplantation technique when performing elective aortic root surgery, mostly in patients with a connective tissue disorder.

\section{References}

1. Kouchoukos NT, Dougenis D. Surgery of the thoracic aorta. $N$ Engl J Med. 1997;336:1876-88.

2. David TE, Feindel CM, Bos J. Repair of the aortic valve in patients with aortic insufficiency and aortic root aneurysm. $J$ Thorac Cardiovasc Surg. 1995;109:345-52.

3. Birks EJ, Webb C, Child A, Radley-Smith R, Yacoub MH. Early and long-term results of a valve-sparing operation for Marfan syndrome. Circulation. 1999;100(suppl):II29-35.

4. Coucke PJ, Willaert A, Wessels MW, Callewaert B, Zoppi N, De Backer J, et al. Mutations in the facilitative glucose transporter GLUT10 alter angiogenesis and cause arterial tortuosity syndrome. Nat Genet. 2006;38:452-7

5. Loeys BL, Chen J, Neptune ER, Judge DP, Podowski M, Holm T, et al. A syndrome of altered cardiovascular, craniofacial, neurocognitive and skeletal development caused by mutations in TGFBR1 or TGFBR2. Nat Genet. 2005;37:275-81. 


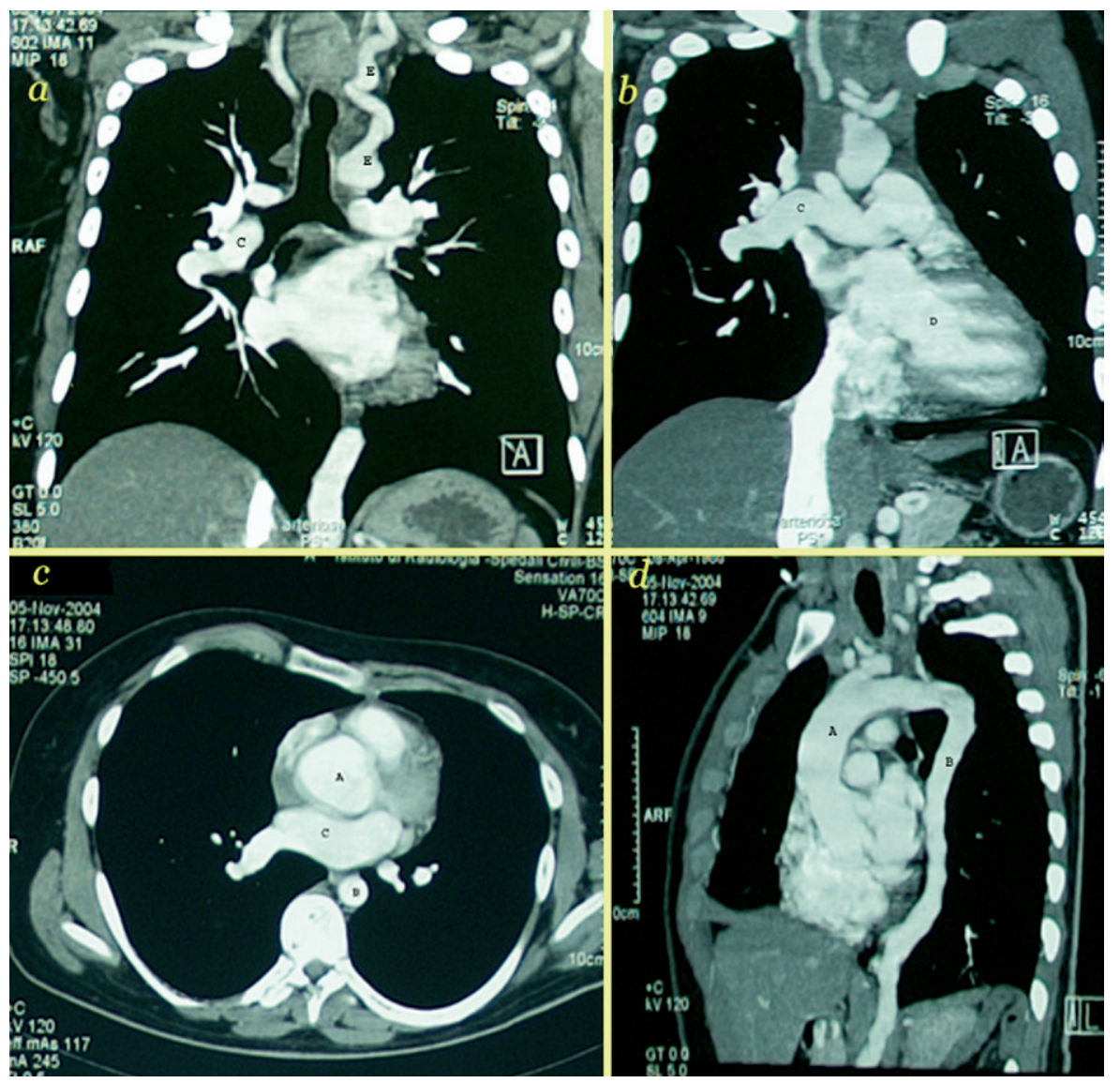

Figure E1. $a$ and $b$, Coronal computed tomographic scan shows an extreme tortuosity of the main pulmonary vessels $(C)$ and left carotid artery (E). D, Left ventricle. c and d, Axial computed tomographic scan shows dilation of the aortic sinus tract $(A)$ and aneurysm of the right pulmonary artery (C). Sagittal view shows narrowing of the descending aorta $(B)$.

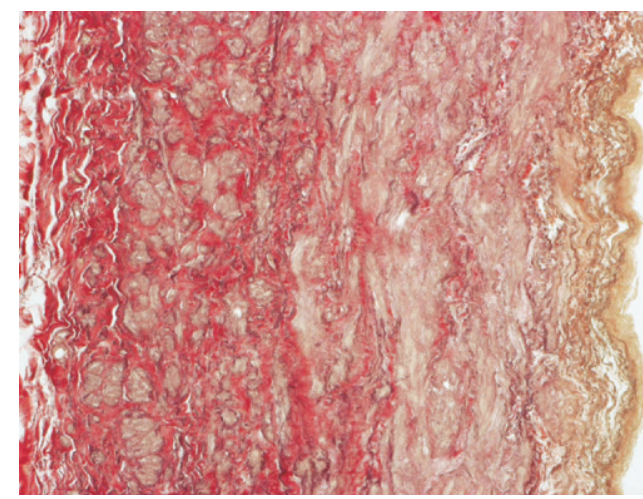

Figure E2. Elastic lamellae are fewer and still tend to be parallel but divide transversely into much more numerous, short, stick-like structures showing small club-like expansions. Note that as a result of the fragmentation, compactness is lost, and the elastic configuration becomes more open and loose. (Weigert van-Gieson stain, original magnification 25×.) 


\section{E References}

E1. Vricella LA, Williams JA, Ravekes WJ, Holmes KW, Dietz HC, Gott VL, et al. Early experience with valve-sparing aortic root replacement in children. Ann Thorac Surg. 2005;80:1622-7.

E2. Heath D, Wood EH, DuShane JW, Edwards JE. The structure of the pulmonary trunk at different ages and in cases of pulmonary hypertension and pulmonary stenosis. J Pathol Bacteriol. 1959;77: 443-56.

E3. Plank L, James J, Wagenvoort CA. Caliber and elastin content of the pulmonary trunk. Arch Pathol Lab Med. 1980;104:238-41. 